\title{
Study Registry Name
}

National Cancer Institute

\section{Source}

National Cancer Institute. Study Registry Name. NCI Thesaurus. Code C94136.

A non-unique textual identifier for the registry. 